\title{
Multi-wavelength emission region of $\gamma$-ray emitting pulsars
}

\author{
S. Kisaka* and Y. Kojima** \\ Department of Physics, Hiroshima University, Higashi-Hiroshima 739-8526, Japan \\ * kisaka@theo.phys.sci.hiroshima-u.ac.jp \\ ** kojima@theo.phys.sci.hiroshima-u.ac.jp
}

\begin{abstract}
Using the outer gap model, we investigate the emission region for the multiwavelength light curve from energetic pulsars. We assume that $\gamma$-ray and non-thermal X-ray photons are emitted from a particle acceleration region in the outer magnetosphere, and UV/optical photons originate above that region. We assume that $\gamma$-rays are radiated only by outwardly moving particles, whereas the other photons are produced by particles moving inward and outward. We parameterize the altitude of the emission region as the deviation from the rotating dipole in vacuum and determine it from the observed multi-wavelength pulse profile using the observationally constrained magnetic dipole inclination angle and viewing angle of the pulsars. We find that the outer gap model can explain the multi-wavelength pulse behavior by a simple distribution of emissivity, and discuss the possibility of further improvement. From observational fitting, we also find a general tendency for the altitude of the $\gamma$-ray emission region to depend on the inclination angle. In particular, the emission region for low inclination angle is required to be located in very low altitude, which corresponds to the inner region within the last-open field line of rotating dipole in vacuum. This model suggests a modification of statistics about observed $\gamma$-ray pulsars. Number of the sources with low inclination and viewing angles increases compared with previous estimate.
\end{abstract}

Subject headings: stars: magnetic field — stars: neutron — pulsars: general

\section{INTRODUCTION}

Pulsars emit over a wide range of energies from radio to $\gamma$-ray. Recent observations by the Fermi Gamma-Ray Space Telescope of more than sixty pulsars (Abdo et al. 2010a; Saz Parkinson et al. 2010) have revealed further details of the structure of the emission region. The detection of the emissions in the GeV energy range from a pulsar magnetosphere means that electrons and positrons are accelerated to more than $\sim 10^{12} \mathrm{eV}$ by the electric field parallel to the magnetic field, which arises in a depleted region of the Goldreich-Julian charge density (Goldreich \& Julian 1969). The light curve in the $\gamma$-ray band is an important tool for probing the particle acceleration and dissipation 
processes in the pulsar magnetosphere, since the maximum energy is determined by the accelerationradiation-reaction-limit for typical energetic pulsars. The $\gamma$-ray emission region has therefore been explored by comparing theoretical models with the observed light curve(e.g., Watters et al. (2009); Venter, Harding \& Guillemot (2009); Romani \& Watters (2010)). The pulsed emission is also detected in other energy bands (X-ray, ultraviolet, optical and radio) for some sources (e.g., Thompson (2004)). The spectral features are non-thermal except for the soft X-ray range, and the light curves from a single object are, in general, different from one energy band to another. For example, profiles of the light curve in one spin period are different in the $\gamma$ - and X-ray ranges in the Vela pulsar (Abdo et al. 2009a). The peak phase of different energy range is expected to coincide, since the emitting particles are related to a pair cascade process. However, the observation shows that the phase depends on the energy bands. This means that their emission regions are not the same region. A complete understanding of light curve behavior in multi-wavelength bands can provide valuable information about the particle acceleration region. Note that we do not discuss soft X-rays, which are believed to be thermal radiation from the neutron star surface (e.g., Jackson et al. (2002)).

Possible origins of non-thermal pulsed emissions have been considered in the polar cap (Daugherty \& Harding 1996), slot gap (Muslimov \& Harding 2004), and outer gap (Cheng, Ho \& Ruderman 1986) models. Recent Fermi observations with high $\gamma$-ray photon number statistics have showed that the phase-averaged spectrum above $200 \mathrm{MeV}$ is well fitted by a power law plus exponential cutoff, and that a cutoff shape sharper than a simple exponential is rejected with high significance (e.g., Abdo et al. (2009a)). This rules out the near-surface emission proposed in polar cap cascade models (Daugherty \& Harding 1996), which would exhibit a much sharp spectral cutoff due to magnetic pair-production attenuation. Thus, pulsed $\gamma$-ray emission originates in the outer magnetosphere, as considered in the outer gap model.

Takata, Chang \& Shibata (2008) (hereafter TCS08) considered a three-dimensional geometrical emission model to fit the observed light curves at different energy bands. The model is extended with some model parameters from the calculated gap structure in a two-dimensional meridian plane. By comparing the light curves of the Vela pulsar, they found that the X-ray emission is produced by both inward and outward emission from the gap region, and that UV/optical emission originates from secondary pairs at a higher altitude. The number of light curves of pulsars observed at $\gamma$-ray and other energy bands is increasing thanks to Fermi, so that it is worthwhile to investigate whether outer gap model is applicable to other sources.

In this paper, we investigate the emission regions of several pulsars by fitting the simplified model of TCS08 to the observed multi-wavelength light curves. In this model, we have to specify the locations of the upper and lower boundaries of the gap region where the non-corotation potential is zero. Therefore, we explicitly introduce the altitude of the gap region as a parameter, in order to fit the observational data easily. The light curves also depend on the dipole inclination and viewing angles. In our method, such parameters are eliminated by other observational data, and only the altitude is changed for the fitting. In the most studies, the lower boundary of the emission region is chosen as the surface of the last-open field lines of the rotating dipole(e.g. Takata, Chang \& Shibata 
(2008); Romani \& Watters (2010)) In this paper, however, the altitude is allowed to be in a wide range in order to explore the possible deviation of magnetic field-line structure from that of a rotating dipole in vacuum. In Section 2, we describe the model assumptions and parameters. In Section 3, we compare the peaks of light curves with those observed at multiple wavelengths, and determine the altitude parameter. Our discussion is presented in Section 4, and lastly, a summary is given in Section 5 .

\section{MODEL DESCRIPTION}

The numerical method for fitting the light curve is well described by Romani \& Watters (2010) and Bai \& Spitkovsky (2010a); but we briefly summarize it in this section to explain one modification. Our model is almost the same as that used by TCS08. However, we explicitly introduce the altitude of the emission region as an additional parameter.

We assume that magnetic field structure is approximately described by a rotating dipole with magnetic moment $\mu$. The angular velocity is $\Omega$, and the magnetic axis is declined by an angle $\alpha$ from the axis of rotation (the z-axis). The magnetic moment changes with time $t$ as

$$
\boldsymbol{\mu}(t)=\mu(\sin \alpha \cos \Omega t \hat{\mathbf{x}}+\sin \alpha \sin \Omega t \hat{\mathbf{y}}+\cos \alpha \hat{\mathbf{z}}) .
$$

The magnetic field produced by the rotating dipole (e.g. Jackson (1975)) can be expressed using the retarded time $t_{r}=t-r / c$ as

$$
\mathbf{B}=-\left[\frac{\boldsymbol{\mu}\left(t_{r}\right)}{r^{3}}+\frac{\dot{\boldsymbol{\mu}}\left(t_{r}\right)}{c r^{2}}+\frac{\ddot{\boldsymbol{\mu}}\left(t_{r}\right)}{c^{2} r}\right]+\hat{\mathbf{r}}\left[\hat{\mathbf{r}} \cdot\left(3 \frac{\boldsymbol{\mu}\left(t_{r}\right)}{r^{3}}+3 \frac{\dot{\boldsymbol{\mu}}\left(t_{r}\right)}{c r^{2}}+\frac{\ddot{\boldsymbol{\mu}}\left(t_{r}\right)}{c^{2} r}\right)\right],
$$

where $r$ is radial distance from the center of the star, and a dot denotes a derivative with respect to $t$.

We assume that radiation direction aligns with magnetic field in a frame rotating with angular velocity $\Omega$ in which the electric field vanishes. Physically, this means that the magnetosphere is filled with a co-rotation enforcing charge. The condition holds only within the light cylinder. The direction of particles in the lab frame is given by

$$
\boldsymbol{\beta}_{0}=\beta_{\|}^{\prime} \hat{\mathbf{B}}+\boldsymbol{\Omega} \times \mathbf{r} / c
$$

where

$$
\beta_{\|}^{\prime}=-\hat{\mathbf{B}} \cdot(\boldsymbol{\Omega} \times \mathbf{r} / c)+\left\{[\hat{\mathbf{B}} \cdot(\boldsymbol{\Omega} \times \mathbf{r} / c)]^{2}-(\boldsymbol{\Omega} \times \mathbf{r} / c)^{2}+1\right\}^{1 / 2},
$$

and $\hat{\mathbf{B}}$ is the unit vector along the magnetic field in lab frame. The particle velocity is highly relativistic, so we have made the approximation $\left|\boldsymbol{\beta}_{0}\right| \rightarrow 1$ in eq. (4). Thus the direction of radiation emitted tangential to the particle velocity vector is given by $\boldsymbol{\beta}_{0}$ in eq. (3). This direction is related to the periodic pulse phase. The observed phase $\phi$ is the sum of the azimuthal angle $\phi_{e m}$ at the 
emission point and the relativistic time delay (Romani \& Yadigaroglu 1995):

$$
\phi=-\phi_{e m}-\frac{\mathbf{r}_{e m} \cdot \hat{\boldsymbol{\beta}_{0}}}{R_{L C}}
$$

where $\mathbf{r}_{e m}$ is the emission point, and $R_{L C}$ the light cylinder radius.

A certain mechanism is needed to fix the lower boundary of the particle acceleration region. In most works, including TCS08, the lower boundary is chosen as the surface of the last-open field lines of a rotating dipole in a vacuum. The field lines are calculated by eq. (2) from the neutron star surface, and the last-open ones are defined as being just tangential to the light cylinder and they form a magnetic surface from the polar cap. The numerical procedure is described by Cheng. Ruderman \& Zhang (2000) in detail and we follow it. In the outer gap model, if particle acceleration occurs in an open zone, the curvature radiation from the accelerated particles forms a narrow cone along the magnetic field lines in a frame rotating with angular velocity $\Omega$. These $\gamma$-ray photons are converted by colliding X-ray photons to $e^{ \pm}$pairs, which tend to screen the accelerating electric field. However, there is no supply of pairs on the last-open field lines and hence no screening of the electric field, since the $\gamma$-ray photons are emitted only toward higher altitudes above the last-open field lines (Cheng. Ho \& Ruderman 1986). The 'real' last-open field lines may be different from ones in a vacuum (Romani 1996; Kojima \& Oogi 2009). We therefore take into account this possible deviation of the boundary. We assume that dipole magnetic field is an approximation within the light cylinder and use eq. (2) as the global magnetic field structure. Even if the overall structure is not different so much, critical value between open and closed field lines is very sensitive to the boundary value at the surface. The "real" last-open field lines do not in general agree with those in vacuum field. Thus we introduce a parameter, altitude of the emission region as a correction factor in order to take into account the deviation of boundary from the vacuum field. In our model this parameter specifies the range of the emission region which is located above or below the last-open field lines within the light cylinder radius. Each different field line originating from the magnetic polar region is parameterized by magnetic colatitudes $\theta_{m}$ and azimuthal angles $\phi_{m}$. Following Cheng. Ruderman \& Zhang (2000), we define open volume coordinates on the polar cap, $\left(r_{o v}, \phi_{m}\right)$, where $r_{o v} \equiv \theta_{m} / \theta_{m}^{p c, 0}\left(\phi_{m}\right)$. The function $\theta_{m}^{p c, 0}$ is the magnetic colatitude of the conventional polar cap angle and generally depends on the magnetic azimuth $\phi_{m}$. The parameter $r_{o v}$ corresponds to the altitude of the emission region: The last-open field lines of a rotating dipole in a vacuum correspond to $r_{o v}=1$, whereas those for higher altitudes have $r_{o v}<1$. Following Takata \& Chang (2009), the maximum value is chosen as $r_{o v}=1.36^{1 / 2}$, which corresponds to the polar cap angle $\theta_{m}^{p c} \sim 1.36^{1 / 2} \theta_{m}^{p c, 0}$, obtained in the force-free limit by Contopoulos, Kazanas \& Fendt (1999). We found that no significant caustics are formed in the sky map, even if the maximum value of $r_{o v}$ is increased.

We assume that the radiation of different energy bands is emitted from different field lines characterized by altitude. The field line relevant to the $\gamma$ - and X-rays is approximated as being the same one. The direction of the emission is tangential to the lines, and inward and outward directions are possible. Both location and direction affect the light curve profile of the energy 
bands. Following the model by TCS08, the $\gamma$-ray radiation above $100 \mathrm{MeV}$ is emitted by particles moving in an outward direction, whereas radiation at lower energy bands is emitted by those moving in both outward and inward directions. We use two conditions to constrain the emission region. First condition is the radial extension of the emission region. The outward emission is restricted to radial distances $r_{n}<R_{L C}$, and the inward one is restricted to $r_{s}<r<\min \left(3 r_{n}, R_{L C}\right)$. The outer boundary $3 r_{n}$ for inward emission comes from the results of dynamic model (TCS08), in which very few ingoing pairs are produced beyond the radial distance $r>3 r_{n}$. Second condition is the azimuthal extension of the emission region. We use the magnetic azimuthal angle of the footprint of field line (i.e., the point where magnetic field line penetrates the neutron star surface) to characterize the field line for given $r_{o v}$. Radial distance to the null charge surface on the field lines significantly depends on the magnetic azimuthal angle. In the outer gap model, most of the pairs are created around the null surface (TCS08). We expect that the gap activity is related to the distance to the null surface. Although the current density should be determined by global conditions, there is no study of the three-dimensional magnetosphere of an inclined rotator. In this paper, we assume that the field lines of both outward and inward emission are active only if the radial distance to null surface $r_{n}$ is shorter than $R_{L C}$. The azimuthal constraint is automatically satisfied for outward emission because the radial extension gives $r_{n}<R_{L C}$. However, for inward emission the condition becomes strong. The radial extension $r_{s}<r<\min \left(3 r_{n}, R_{L C}\right)$ allows for the regions $r<R_{L C}$ on the field lines with $r_{n}>R_{L C}$. They are not active, so that the corresponding regions should be excluded. The critical value $3 r_{n}$ was obtained by fitting to Vela pulsar (TCS08). It is not straightforward to apply it to other sources. The mean free path $\lambda(r)$ of the pair creation process between the $\gamma$-ray and thermal X-ray emissions from the stellar surface is estimated as $\lambda(r) \sim 5.6 P^{13 / 21}\left(B_{s} / 10^{12} G\right)^{-2 / 7} r$ (Tang et al. 2008). The value at the null pointis $\lambda\left(r_{n}\right)$ found to be in a range of $(2-3) r_{n}$ for our samples. Our light curves especially peak positions are not changed even by adopting $2 r_{n}$ as the outer boundary for inward emission.

Spatial distribution of the emissivity is approximated by the step function-type, but the peak positions weakly depend on the detailed emissivity distribution.

We assume that the overall structure of the light curve comes not from the emissivity distribution, but from a bunch of many field lines in the observation, that is, caustics. The appearance of caustics strongly depends on the observational viewing angle $\xi$ and the intensity distribution. In this paper, we focus on the peak phases of the light curve, so we adopt a simple, uniform emissivity along all magnetic field lines, which is independent of both the magnetic azimuthal angle $\phi_{m}$ and the altitude $r_{o v}$. The fitting does not completely reproduce the observations so, in Section 4, a simple improvement to the emissivity distribution is considered which leads to a much better fit.

We now explain our fitting method. For fixed inclination angle $\alpha$ and viewing angle $\xi$, the light curve as a function of phase $\phi$ depends only on the altitude $r_{o v}$. The intensity is calculated in the range $r_{o v}<1.36^{1 / 2}$ with a bin width of 0.02 . There are no significant caustics for large $r_{o v}$.

In the observed light curve, the reference phase $\phi=0$ is assumed to be located at the radio 
emission peak maximum in most studies (e.g., Abdo et al. (2010a)). However in the model light curve, the conventional reference phase $\phi=0$ occurs when the magnetic axis, spin axis and Earth line of sight lie all in the same plane. These two reference phases do not agree with each other since it is generally assumed that radio emissions arise at non-zero altitude in most empirical studies. Following Romani \& Watters (2010), we allow a shift by $-0.1 \leq \delta \phi \leq 0.1$ in the model reference rotation phase. This degree of freedom does not significantly affect the determination of the altitude parameter $r_{o v}$, because we use the peak separation in the observed $\gamma$-ray and X-ray light curves which are emitted at the same $r_{o v}$. For the sources showing a double-peak pulse shape in the observed $\gamma$-ray light curve, we use the peak separation. For those showing a single-peak, we use the phase separation between the $\gamma$-ray peak and one of X-ray peaks. This is the benefit of considering $\gamma$-ray and X-ray light curves simultaneously. Subsequently, we look for the altitude of the UV/optical emission region using the $\gamma$-ray upper limit of $r_{o v}$.

\section{RESULTS}

In this section, we compare our model with pulse profiles observed at multiple wavelengths for seven pulsars. The sources are chosen using two criteria. One is that non-thermal pulses are detected in addition to the $\gamma$-ray and radio bands. Our concern is to explore whether or not the emission region for different energy bands is explained by the TCS08 model. The second criterion is that the geometrical parameters, $\alpha$ and $\xi$ are observationally constrained by the relativistic Doppler-boosted X-ray pulsar wind nebula (PWN) (Ng \& Romani 2008) or radio polarization data (e.g. Lyne \& Manchester (1988)). The torus fitting method constrains the viewing angle $\xi$ only. A small allowed range of $|\alpha-\xi| \leq 10^{\circ}$ is assumed for samples in which only $\xi$ is constrained due to the fact that radio emission from the pulsar polar region is detected. The geometrical parameters for the pulsars are listed in Table 1. We use these values, although there are some uncertainties in them. The results are summarized in Figs. 1 and 2, Fig. 1 shows the intensity map for outward (upper panel) and inward (lower panel) emission as a function of the altitude of the emission region $r_{o v}$ and rotational phase. The upper and lower panels in Fig. 2 are their pulse profiles for outward and inward emission in $\gamma$-ray and $\mathrm{X}$-ray emission regions.

\subsection{Vela pulsar (PSR J0835-4510)}

We start with the Vela pulsar, which has been well studied to test the validity of our simple model. TCS08 considered this source, but they used geometric parameters that are slightly different from ours.

Pulse profiles have been detected in optical to $\gamma$-ray bands. The observed pulse profile in $\gamma$-ray

band by Fermi (Abdo et al. 2010b) shows a prominent double-peak structure and bridge emission between the two peaks. The first and second peaks are located at the phases $\phi \sim 0.13$ and $\phi \sim 0.56$, 
respectively, and the separation is $\Delta \phi=0.43$. We show the intensity map for outward emission as a function of the altitude of the emission region and rotation phase in upper panel of Fig. 1(A). The emission altitude producing a peak separation $\Delta \phi=0.43$ is $r_{o v} \sim 1.05-1.06$.

The X-ray data from RXTE (Harding et al. 2002) also shows a double-peak structure but the second peak broadens toward early phase. The calculated intensity map is shown for outward and inward emissions in the upper and lower panels of Fig. 1(A). The main double peaks are located at the same phases as those in the $\gamma$-ray band, so they are interpreted as being formed by outward emission. The broad component before the second peak at $\phi \sim 0.47$ is associated with the caustic formed by the inward emission, as shown in the lower panel. We attempted a fit without the inward emission, but found that the inward emission is needed in order to reproduce the observed X-ray pulse profile. The necessity of inward emission was discussed in TCS08. Thus, the peak positions of $\gamma$ - and X-ray pulses can be explained with the same value of $r_{o v} \sim 1.05-1.06$. The contour map, however, shows a minor peak at $\phi \sim 0.8$ formed by the outward emission. The peak was not observed in the $\gamma$-ray band.

We compare our model with the UV data of Romani, Kargaltsev \& Pavlov (2005) and optical

data of Gouiffes (1998). The pulse profiles in both bands are very similar, that is, they have a double-peak structure at the same phases. The peak phases however differ from those of the $\gamma$ and X-ray bands. The first peak of the UV/optical bands shifts to a later phase $\phi \sim 0.27$ and the second peak shifts to an earlier phase $\phi \sim 0.46$, so that the peak separation becomes smaller. It can be seen from Fig. 1(A) that such a double-peak structure corresponds to $r_{o v} \sim 0.65-0.80$ for the outward emission. The corresponding inward emission cannot be detected since its observable range is $r_{o v} \geq 0.9$, as shown in the lower panel. The choice of outward emission with $r_{o v} \sim 0.65-0.80$ is also supported by the fact that the second peak at $\phi \sim 0.46$ in the UV/optical ranges is sharper than the first one at $\phi \sim 0.27$, because of their different dependence on $r_{o v}$. Thus, we have reproduced the pulse profiles of optical to $\gamma$-ray bands by the caustics model without any detailed assumptions about emissivity. From the fitting model, we found three conditions for the emission region. (1) The UV/optical emission region is located at an altitude above the $\gamma$ - and X-ray emission region of $r_{o v} \sim 1.05-1.06$. (2) There is a separation of altitude between the X-ray and optically dominant emission regions. (3) The UV/optical emission range, $\Delta r_{o v} \sim 0.15$, is broader than that of $\gamma / \mathrm{X}$-ray emission regions, $\Delta r_{\text {ov }} \sim 0.02$.

\subsection{PSR J0659+1414}

The pulsar PSR J0659+1414 has also been observed in the $\gamma$-ray to optical bands. We use the $\gamma$-ray data from Fermi (Weltevrede et al. 2010), X-ray data from XMM-Newton (De Luca et al. 2005), UV data from Shibanov et al. (2005) and optical data from Kern et al. (2003). The Xray data is a combination of thermal (blackbody) and non-thermal (power-law) emissions and is consistent with a cooling middle-aged neutron star (e.g., Becker \& Trüper (1997)). At soft Xrays, the pulse fraction is low and the pulsations are sinusoidal, as is typical for thermal emissions 
from the surface of a neutron star with non-uniform temperature distribution. At higher energies $(>1.5 \mathrm{keV})$, where the non-thermal component dominates, the pulsed fraction increases and the profile becomes single peaked. We, therefore, consider the pulse profiles of hard X-rays $(>1.5 \mathrm{keV})$ only.

The pulse profile in the $\gamma$-ray band shows a relatively broad single peak, which lags the radio maximum peak by $\phi \sim 0.2$ in phase. The peak in the non-thermal X-ray pulse is at $\phi \sim 0.7-0.8$, which is different from the phase of the $\gamma$-ray peak. This phase difference cannot be ignored, although the peaks in the $\gamma$ - and X-ray data are rather broad, and hence the difference may be diminished somewhat by including the phase error. We interpret these pulse profiles as being emissions at different phases and different directions: the peak of $\gamma$-ray is formed by outward emission, whereas that of X-ray is formed by inward emission. The intensity maps are given in the upper panel (outward emission) and lower panel (inward emission) of Fig. 1(B), respectively. From this figure, we see that a peak separation $\Delta \phi=0.55$ between the $\gamma$-ray and X-ray data corresponds to an emission altitude of $r_{o v} \sim 1.13-1.14$ by shifting the reference phase by $\delta \phi=0.06$ to an earlier phase. The emission altitude cannot be fixed without the X-ray data: a shift of peak phase is allowed, so $r_{o v}$ is unknown. This ambiguity is removed by considering multi-wavelength light curves. From the intensity map, we expect another very sharp caustic at $\phi \sim 0.65$, but there is no counterpart in the $\gamma$-ray observations (top panel of Fig. 4 in Weltevrede et al. (2010)). We discuss this missing peak in Section 4.2. In the lower right panel of Fig. 4 in Weltevrede et al. (2010), the light curve for inward emission is given. The X-ray data may be a combination of inward and outward emissions, but the X-ray profile observed by De Luca et al. (2005) is similar to that of inward emission only. This means phenomenologically that outward emission of X-rays is weak in this source.

The pulse profiles in the UV (Shibanov et al. 2005) and optical bands (Kern et al. 2003) are almost the same shape and have a clear double-peak structure unlike the single peak in the $\gamma$ and X-ray bands. The first peak at $\phi \sim 0.02$ is later than the $\gamma$-ray peak and the second peak at $\phi \sim 0.10$ is later phase than the X-ray peak. From the upper panel of Fig. 1(B), we see that the observed first peak can be reproduced by outward emission at an altitude $r_{o v}<1.10$. Here we have a weak condition because the peak position depends only weakly on $r_{o v}$. For the second peak, the inward emission forms caustics for $0.90<r_{o v}<1.04$. Thus, the altitude of the emission region in the UV/optical bands is identified as $r_{o v} \sim 0.90-1.04$, where the lower limit is set by a coarse bin of the phase in the observational data.

Kern et al. (2003) have already investigated the multi-wavelength light curve of this pulsar using a similar method to ours, but could not explain the profile using geometrical parameters which are consistent with radio polarization data (Everett \& Weisberg 2001). The reason for this is that the lower boundary of the emission region was chosen as the last-open field lines in the vacuum dipole field, that is, $r_{o v}=1.0$. In our analysis, by allowing $r_{o v} \geq 1.0$, the phase of peaks can be successfully fitted by using observed geometrical parameters. This suggests that the actual lower boundary of the gap is slightly different from the last-open field lines in a vacuum dipole. 


\subsection{PSR J0205+6449}

The $\gamma$-ray pulse profile observed by Fermi (Abdo et al. 2009b) shows a double-peak structure. The first peak is offset from the radio peak by $\phi \sim 0.08$, and the second is at $\phi \sim 0.57$. The separation is $\Delta \phi=0.49$. The X-ray data from $R X T E$ (Livingstone et al. 2009) are consistent with the results from $X M M$-Newton (Kuiper et al. 2010). The spectral shape can be fitted by a power law such that most of the emission is non-thermal and the thermal component is constrained by the upper limit (Kuiper et al. 2010). The observed X-ray pulse profile shows two peaks aligned in phase over a wide energy range of $\sim 0.5-270 \mathrm{keV}$, and is also very similar to that of the $\gamma$-ray band.

We show the intensity maps for outward and inward emission in the upper and lower panels of Fig. 1(C), respectively. As seen in the upper panel, the emission altitude for the double-peak with $\Delta \phi=0.49$ is $r_{o v} \sim 0.97-0.98$. A shift of the reference phase $\delta \phi$ is not necessary in this source. As argued in TCS08, outward emission dominates in the light curve for a young pulsar with a strong non-thermal X-ray component, like the Crab pulsar. PSR J0205+6449 is the youngest pulsar in our sample (characteristic age $\tau_{c} \sim 5 \times 10^{3} \mathrm{yr}$ ) and shows rather strong non-thermal radiation in the X-ray band. Thus, it is likely that only outward omission contributes to the observed X-ray light curve in this pulsar.

\subsection{PSR J2229+6114}

The light curve for $\gamma$-rays observed by Fermi (Abdo et al. 2009c) shows an asymmetric single peak at $\phi \sim 0.49$. The tail extends down to $\phi \sim 0.2$. The peak position depends slightly on the energy range above $100 \mathrm{MeV}$, but the amount of shift is only $\sim 0.04$. The X-ray pulse profile observed by XMM-Newton (Abdo et al. 2009c) shows a double-peak structure. No peak is seen at the $\gamma$-ray peak phase. The separation between first X-ray peak and the $\gamma$-ray peak is $\Delta \phi \sim 0.32$, and the separation between the second X-ray peak and the $\gamma$-ray peak is $\Delta \phi \sim 0.14$.

The intensity map for outward emission is shown in the upper panel of Fig. 1(D). We consider the formation of the $\gamma$-ray peak and two X-ray peaks as being due to outward emission only. Such a solution is possible by choosing an emission altitude of $r_{o v} \sim 1.01-1.02$ with a small phase shift $\delta \phi=0.03$. The intensity map for inward emission shows a sudden decrease in the number counts for $r_{o v}<1.06$. The peak becomes broad and hence the contribution of the inward emission is not very important. We show the light curve of inward and outward emission for $r_{o v} \sim 1.01-1.02$ in Fig. 1(D). The outward emission curve is very similar to the observations in the $\gamma$ - and X-ray bands.

In this pulsar, we need to use all the light curves simultaneously in order to determine the range of $r_{o v}$. Since emissions with smaller values of $r_{o v}$ are not seen, if $\gamma$-ray and optical emission regions are separated $\Delta r_{o v}>0.10$, similar to the Vela pulsar and PSR J0659+1414, we predict that an optical pulse profile cannot be observed or will be only very weakly detected. This is consistent 
with the fact there have been no reports of the detection of a pulse in the lower energy band for this pulsar.

\subsection{PSR J1420-6048}

The $\gamma$-ray light curve from Fermi (Weltevrede et al. 2010) shows a broad peak at $\phi \sim 0.2-0.5$. This peak may consist of two components, but it is not clear in the current photon statistics. The X-ray pulse profile from $A S C A$ is detected weakly at a marginal level, and shows a broad peak at $\phi \sim 0.6-0.7$, which is different from the $\gamma$-ray peak (Roberts. Romani \& Johnston 2001; Becker 2009). Recently, in the table of Marelli, De Luca \& Caraveo (2011) they list this object as non-thermal dominated source in X-ray. The pulsed X-ray profile is likely to originate from the non-thermal component.

Since the light curve of this pulsar and its geometrical parameters are similar to those of PSR J0659+1414, we adopt the same interpretation. That is, the $\gamma$-ray peak is formed by outward emission, whereas the X-ray peak is formed by inward emission. From the intensity map in Fig.11(E), an emission altitude of $r_{o v} \sim 1.10-1.11$ corresponds to one broad peak at $\phi \sim 0.2-0.5$ by outward emission and another at $\phi \sim 0.6-0.7$ by inward emission. Here a small shift $\delta \phi=0.10$ toward earlier phase is used. Since there are similarities in both the $\gamma$ - and X-ray light curves and the geometrical parameters between this pulsar and PSR J0659+1414, we expect a similar double-peak pulse profile in the optical band, if it is detected.

\subsection{PSR J2021+3651}

Observations in the $\gamma$-ray band have been obtained by Fermi (Abdo et al. 2009d) and AGILE (Halpern et al. 2008). The observed light curve shows a sharp double-peak structure. The first peak is offset from the radio peak by $\phi \sim 0.16$ and the two peaks are separated by $\Delta \phi \sim 0.47$. The X-ray light curve in Abdo et al. (2009d) shows a relatively sharp peak associated with first peak in the $\gamma$-ray light curve albeit with weak photon statistics. In this paper, we assume that at least the first peak is non-thermal in origin. The possible contribution of non-thermal X-ray emissions is also discussed in Hessels et al. (2004) and Van Etten. Romani \& Ng (2008). We expect that this assumption will be tested by phase-resolved spectra from future observations.

As seen in the upper panel of Fig. 1(F), the emission altitude is $r_{o v} \sim 0.97-0.98$, for which there is a $\gamma$-ray double-peak profile with separation $\Delta \phi=0.47$ and a relative shift $\delta \phi=0.06$ toward later phase. The peak of non-thermal emission at $\phi=0.15-0.20$ in the X-ray light curve is found to be formed by outward emission only. The relatively weak second peak in the X-ray band is consistent with the case of PSR J0205+6449. Thus, the three model parameters for this pulsar and PSR J0205+6449 are very similar, as shown in Table 1 . 


\subsection{PSR J1057-5226}

The $\gamma$-ray light curve from Fermi (Abdo et al. 2010c) shows a broad peak at $\phi \sim 0.25-0.65$. This probably consists of two components, but it is not clear. De Luca et al. (2005) extract only a power-law component of the X-ray light curve and their Fig. 13 shows a two peaks at $\phi \sim 0.2-0.3$ and $\phi \sim 0.9-1.0$, although the data are very coarse. We regard the light curve as being produced by a non-thermal X-ray component.

The observed light curve may be regarded either as a broad peak consisting of weak peaks and a relatively bright bridge emission or as a result of the range of the emission region widening towards lower altitudes. In the latter interpretation the fitted $r_{o v}$ is only a lower limit. We thus focus on the width of the $\gamma$-ray peak. From the upper panel of Fig. 1(G), the emission altitude

is $r_{o v} \sim 0.93-0.94$ Even if we assume a double-peak structure with the first peak at $\phi \sim 0.31$ and the second peak at $\phi \sim 0.59$ following Abdo et al. (2010c), we have $r_{o v} \sim 0.90-0.91$, which is very similar to the value obtained above. Thus we have $r_{o v} \sim 0.90-0.95$ in either case. The phase shift is $\delta \phi=0.10$ in this pulsar. The first peak in the X-ray light curve is formed by outward emission, but the second one cannot be produced for the same altitude. This may be a drawback to our model, but the present X-ray data are coarse and a much more precise non-thermal X-ray light curve is needed.

\section{DISCUSSION}

\subsection{Statistical properties of the emission region}

In the previous section, we have shown that the peak phases of seven pulsars emitting $\gamma$ - and X-rays can be successfully fitted using the TCS08 outer gap model, in which both $\gamma$-rays and X-rays originate from the same magnetic field line characterized by an altitude $r_{o v}$. The parameter $r_{o v}>1$ is needed in the light curve fitting for some sources. Moreover, the inclusion of inward emission for $\mathrm{X}$-rays causes a variety of pulse profiles in both bands. The parameter $r_{o v}$ could not be determined solely using $\gamma$-ray data for a single $\gamma$-ray peak pulsar. But, by considering the X-ray light curve, the parameter is uniquely determined for PSRs J0659+1414, J2229+6114 and J1420-6048.

It is worthwhile to explore the general dependence of the altitude $r_{o v}$ on other characteristics if any, although there may not be enough data for a proper statistical analysis. In Fig. 3, $r_{o v}$ is plotted as a function of inclination angle $\alpha$, spin-down luminosity $L_{s d}$, characteristic age $\tau_{c}$ and surface dipole magnetic field $B_{s}$. We found that there is a significant correlation between $r_{o v}$ and the inclination angle $\alpha$ only; the relations of $r_{o v}$ with the other parameters are very weak. This correlation suggests that the deviation from a vacuum rotating dipole field is large for small inclination angle. It is very interesting to compare this result with that in a force-free magnetosphere. Bai \& Spitkovsky (2010b) proposed that the separatrix layer at an altitude of 0.90-0.95 times the height of the last-open field line is relevant to emissions in a three-dimensional inclined force-free 
magnetosphere. This altitude, which is not exactly symmetric with respect to the magnetic azimuthal angle $\phi_{m}$, but can be approximated by the value at $\phi_{m}=0$, is plotted in Fig. 3 as purple downward and blue upward triangles. Two linear fitting lines are also shown. The altitude $r_{o v}$ decreases with the inclination angle $\alpha$ in both our model and the separatrix layer model of a force-free magnetosphere. However, the emission region in the separatrix layer model extends even outside the light-cylinder, whereas ours is well localized around null points. Accounting for this difference may be important for further improvement of the model of the emission region based on a force-free magnetosphere.

The thickness of the gap region, $w$, is not known, but it is sometimes assumed to decrease with the spin-down luminosity $L_{S D}$ (Watters et al. 2009; Romani \& Watters 2010). We have $w=1-r_{o v}$, if the lower boundary of the gap is fixed as the last-open field line in the vacuum dipole field. This assumption is tested in the lower left panel of Fig. 3, in which the relation $\left(1-r_{o v}\right) \approx$ $\left(L_{S D} / 10^{33} \text { ergs }^{-1}\right)^{-1 / 2}$ is plotted as a light green curve. (The curve is not fitted to the data points.) This suggests that the assumption of maximum altitude, $r_{o v}=1.0$, is not a good one. This discovery affects expected number of the $\gamma$-ray pulsars in the observation. From geometrical reason, the pulsed emission by caustics is limited to a certain range between inclination and viewing angles.

Romani \& Watters (2010) showed the range of observable pulsars with $r_{o v}=0.95,0.90$ and 0.70 for outer gap model in their Fig. 16. We recalculate it and show the result in Fig. 4. The observable range of viewing angle $\xi$ is below the curves. Our finding in Fig. 3 is that $r_{o v}$ is a function of the inclination angle, which is similar to that of the separatrix layer model. We also show the observable range by the empirical relation obtained in Fig. 3 as black solid line, for which the altitude is chosen as 0.925 times the height of the last-open field line in force-free magnetosphere. The figure shows that sources with low inclination and viewing angles become observable. For example, pulsar with the inclination angle $\alpha=30^{\circ}$ can be detected for $\xi>60^{\circ}$ for $r_{o v}=0.95$, but for $\xi>30^{\circ}$. Thus expected number increases approximately twice for sources with the low inclination and viewing angles.

\subsection{The phenomenological limitation for emissivity}

The caustic model considered in Section 3 provides peak positions consistent with observation, but there are also some additional, unseen peaks. These are interpreted as being prohibited by some mechanism. In this section, we consider an improvement to our model that takes into account a very simple distribution for the emissivity. Detectable $\gamma$-rays are radiated with large multiplicity by the pair plasma in the gap region. Therefore, the mean free path of a $\gamma$-ray photon should be less than light cylinder radius (Takata \& Chang 2007). The pair creation mean free path is given by $\lambda(r) \sim 5.6 P^{13 / 21}\left(B_{s} / 10^{12} G\right)^{-2 / 7} r$ (Tang et al. 2008) for an assumed limiting distance to the null point $r_{n, \mathrm{lim}}$. The position of the null point of inclined pulsars, where the accelerating electric field arises, depends significantly on magnetic azimuthal angle, so the intensity of $\gamma$-ray emission 
also depends on the magnetic azimuthal angle. Therefore, active field lines should be limited in the azimuthal direction. By taking into account the azimuthal extensions with $\lambda\left(r_{n, l i m}\right) \lesssim 0.2-0.7 R_{L C}$ listed in Table 1, the fits of the resultant light curves, which are shown in Fig. 5 , become better. In the same figure, we also show the radial distance to the emission points of the observed photons against the rotation phase. Note that, for the Vela pulsar, the minor third peak at $\phi \sim 0.8$ still remains even after the inclusion of the azimuthal extension limit. The corresponding radial distance of emission points is relatively large, so that the photon energy is expected to be soft. The third peak is not observed in the $\mathrm{GeV}$ band, but may appear in a much lower energy band. At least, the minor third peak of the X-ray light curve appears to be associated with the same caustic.

We have also tried to improve the X-ray light curve with some other simple assumptions, but have not had good results. The reason for this is that there are many ways for X-ray emitting particles to be created: via thermal, magnetospheric emissions and magnetic pair creation. Therefore, the three-dimensional effect of the propagation of $\gamma$-ray photons and soft X-ray photons is very important. Without it we cannot successfully explain the light curve.

\subsection{The location of the UV/optical emission region}

We explored the UV/optical emission region for Vela and PSR J0659+1414. The results are qualitatively similar: the altitude range of UV/optical emission, $\Delta r_{o v} \sim 0.15$, is broader than that for $\gamma$ - and X-rays, $\Delta r_{o v} \sim 0.02$; and both emission regions are not continuous and connected, but are widely separated. The separation may come from two competing mechanisms: a decrease of emissivity and an increase of synchrotron intensity in the UV/optical bands with altitude.

As discussed in TCS08, outward emission is generally dominant in UV/optical emission, as shown in their Fig. 4. The explanation is the following. The number of created pairs is the main cause of the difference between inward and outward emissions in the UV/optical bands, since the collision angles with magnetospheric X-rays are not different for outgoing and ingoing $\gamma$-ray photons in the acceleration region. More outgoing $\gamma$-rays are emitted, and hence more outgoing secondary pairs are produced. Thus, the synchrotron emission for outgoing secondary pairs produced by magnetic X-rays is brighter than that for the ingoing secondary pairs. The observed flux strongly depends on the geometrical configuration. The outward emission in the UV/optical bands may not point toward us even though the intrinsic emission is strong. Our results show that the peaks in the Vela pulsar can be explained by outward emission alone, while those in PSR J0659+1414 require both inward and outward emissions. Our result suggests that outward emission is significantly suppressed in PSR J0659+1414, to the level of the intrinsically weak inward emissions. The stronger component is hidden, because the observable altitude range is narrow, as shown in Fig. 1(B). This may explain the fact that UV/optical flux is smaller than the value extrapolated from non-thermal

X-rays, as seen Fig. 4 of Mignani et al. (2010), whereas the flux coincides with the extrapolation in the Vela pulsar. This interpretation may be tested in PSR J1057-5226. We also suggest that this difference is the reason why PSR J0659+1414, which has similar geometrical parameters to 
the Vela pulsar, has an observable optical spectrum (Mignani, Pavlov \& Kargaltsev 2010), and the flux is slightly smaller than the extrapolation from non-thermal X-ray emission. The pulse profile has not yet been determined, but the peaks should appear at a phase $0.3<\phi<0.6$ and be due to outward emission.

\section{SUMMARY}

We have calculated the light curves of emissions using the TCS08 outer gap model and compared them with observed multi-wavelength light curves. We find that the model can successfully explain the peak positions of multi-wavelength light curves. In order to determine the altitude of the emission region, the observed X-ray light curve is important, especially when there is a single peak in the $\gamma$-ray light curve.

The fit of a light curve based on a simple emissivity distribution can be improved by taking into account the limitation of azimuthal extension in which a reasonable value of the $\gamma$-ray photon mean free path is adopted. The resulting difference between model and observed $\gamma$-ray light curves becomes small; however, there may still be an unseen peak, such as the minor third peak in Vela.

The best-fit values of the altitude of the emission region for PSRs J0659+1414 and J1420-6048, suggest a deviation from the last-open field lines of a vacuum dipole field. The real last-open field lines lie inside those of vacuum dipole field, $r_{o v}<1.0$. This shift suggests that the lower boundary is very similar to that of a force-free magnetosphere. We find that the altitude of the emission region is correlated with inclination angle. This relationship is also very similar to that in a force-free magnetosphere. The lower boundary of emission region has been assumed to $r_{o v}=1$ so far, but our model fits do not support it. This modification of the boundary of the magnetosphere suggests that the pulsars with low inclination and viewing angles are likely to be detectable. Thus the expected number in the future observation in the previous works(Takata, Wang \& Cheng (2011); Watters \& Romani (2011)) is underestimated for the sources with low inclination and viewing angles.

\section{Acknowledgements}

The authors thank S. Shibata and J. Takata for much valuable discussion. This work was supported in part by the Grant-in-Aid for Scientific Research from the Japan Society for Promotion of Science(S.K.) and from the Japanese Ministry of Education, Culture, Sports, Science and Technology(Y.K. No.21540271). 


\section{REFERENCES}

Abdo, A. A., et al. 2009a, ApJ, 696, 1084

Abdo, A. A., et al. 2009b, ApJ, 699, L102

Abdo, A. A., et al. 2009c, ApJ, 706, 1331

Abdo, A. A., et al. 2009d, ApJ, 700, 1059

Abdo, A. A., et al. 2010a, ApJS, 187, 460

Abdo, A. A., et al. 2010b, ApJ, 713, 154

Abdo, A. A., et al. 2010c, ApJ, 720, 26

Bai, X.-N., \& Spitkovsky, A. 2010a, ApJ, 715, 1270

Bai, X.-N., \& Spitkovsky, A. 2010b, ApJ, 715, 1282

Becker, W., 2009, in Becker W., ed., Astrophys. Space Sci. Libr. Vol. 357, Neutron Stars and Pulsars. Springer, Berlin, p.133

Becker, W., \& Trümper, J. 1997, A\&A, 326, 682

Cheng, K. S., Ho, C., \& Ruderman, M. 1986, ApJ, 300, 522

Cheng, K. S., Ruderman, M. A., \& Zhang, L. 2000, ApJ, 537, 964

Contopoulos, I., Kazanas, D., \& Fendt, C. 1999, ApJ, 511, 351

Daugherty, J. K., \& Harding, A. K. 1996, ApJ, 458, 278

De Luca, A., Caraveo, P.A., Mereghetti, S., Negroni, M., \& Bignami, G. F. 2005, ApJ, 623, 1051

Everett, J. E., \& Weisberg, J. M. 2001, ApJ, 553, 341

Goldreich, P., \& Julian, W. H. 1969, ApJ, 157, 869

Gouiffes, C. 1998, in Neutron Stars and Pulsars, ed N. Shibazaki et al. (Tokyo: Univ. Acad. Press), 363

Halpern, J. P., et al. 2008, ApJ, 688, 33

Harding, A. K., Strickman, M. S., Gwinn, C., Dodson, R., Moffet, D., \& McCulloch, P. 2002, ApJ, 576,376

Hessels, J. W. T., Roberts, M. S. E., Ransom, S. M., Kaspi, V. M., Romani, R. W., Ng, C.-Y., Freire, P. C. C., \& Gaensler, B. M. 2004, ApJ, 612, 389 
Jackson, J. D. 1975, Classical Electrodynamics (2nd ed.; New York: Wiley)

Jackson, M. S., Halpern, J. P., Gotthelf, E. V., \& Mattox, J. R. 2002, ApJ, 578, 935

Johnston, S., Karastergiou, A., \& Willett, K. 2006, MNRAS, 369, 1916

Kern, B., Martin, C., Mazin, B., \& Halpern, J. P. 2003, ApJ, 597, 1049

Kojima, Y., \& Oogi, J. 2009, MNRAS, 398, 271

Kuiper, L., Hermsen, W., Urama, J. O., den Hartog, P. R., Lyne, A G., \& Stappers, B. W. 2010, A\&A, 515, A34

Livingstone, M. A., et al., 2009, ApJ, 706, 1163

Lyne, A. G., \& Manchester, R. N. 1988, MNRAS, 234, 477

Marelli, M., De Luca, A., \& Caraveo, P. A. 2011, ApJ, 733, 82

Mignani, R. P., Pavlov, G. G., \& Kargaltsev, O. 2010, ApJ, 720, 1635

Mignani, R. P., Sartori, A., De Luca, A., Rudak, B., Słowikowska, A., Kanbach, G., \& Caraveo, P. A. 2010, A\&A, 515, A110

Muslimov, A. G., \& Harding, A. K. 2004, ApJ, 606, 1143

Ng, C.-Y., \& Romani, R. 2008, ApJ, 673, 411

Roberts, M. S. E., Romani, R. W., \& Johnston, S. 2001, ApJ, 561, L187

Romani, R. W. 1996, ApJ, 470, 469

Romani, R. W., \& Watters, K. P. 2010, ApJ, 714, 810

Romani, R. W., Kargaltsev, O., \& Pavlov, G. G. 2005, ApJ, 627, 383

Romani, R. W., \& Yadigaroglu, I.-A. 1995, ApJ, 438, 314

Saz Parkinson, P. M., et al. 2010, ApJ, 725, 571

Shibanov, Y. A., Sollerman, J., Lundqvist, P., Gull, T., \& Lindler, D. 2005, A\&A, 440, 693

Takata, J., \& Chang, H.-K. 2007, ApJ, 670, 692

Takata, J., \& Chang, H.-K. 2009, MNRAS, 392, 400

Takata, J., Chang, H.-K., \& Shibata, S. 2008, MNRAS, 386, 748 (TCS08)

Takata, J., Wang, Y., \& Cheng, K. S. 2011, ApJ, 726, 44 
Tang, A. P. S., Takata, J., Jia, J. J., \& Cheng, K. S. 2008, ApJ, 676, 562

Thompson, D. J. 2004, in Cosmic Gamma Ray Sources, ed. K. S. Cheng \& G. E. Romero (Dordrecht: Kluwer), 149

Van Etten, A., Romani, R. W., \& Ng, C.-Y. 2008, ApJ, 680, 1417

Venter, C., Harding, A. K., \& Guillemot, L. 2009, ApJ, 707, 800

Watters, K. P., Romani, R. W., Weltevrede, P., \& Johnston, S. 2009, ApJ, 695, 1289

Watters, K. P. \& Romani, R. W. 2011, ApJ, 727, 123

Weltevrede, P., \& Johnston, S. 2008, MNRAS, 391, 1210

Weltevrede, P., \& Wright, G. 2009, MNRAS, 395, 2117

Weltevrede, P., et al. 2010, ApJ, 708, 1426

Zhang, L., \& Cheng, K. S. 1997, ApJ, 487, 370 
TABLE 1 Pulsar parameters

\begin{tabular}{|c|c|c|c|c|c|c|c|c|c|c|}
\hline Name & $\begin{array}{c}\log \left(L_{S D}\right) \\
\left(\operatorname{erg~s}^{-1}\right) \\
(2)\end{array}$ & $\begin{array}{c}\tau_{c} \\
(\mathrm{kyr}) \\
(3)\end{array}$ & $\begin{array}{c}\log \left(B_{s}\right) \\
(\mathrm{G}) \\
(4)\end{array}$ & $\begin{array}{c}\alpha \\
(\text { degrees) } \\
(5)\end{array}$ & $\begin{array}{c}\xi \\
\text { (degrees) } \\
(6)\end{array}$ & Reference & $\begin{array}{c}r_{o v}\left(\gamma^{-}, \mathrm{X}-\text { ray }\right) \\
(8)\end{array}$ & $\begin{array}{c}r_{\text {ov }}(\mathrm{UV} / \text { optical }) \\
(9)\end{array}$ & $\begin{array}{c}r_{n, l i m} \\
\left(R_{L C}\right) \\
(10)\end{array}$ & $\begin{array}{c}\lambda\left(r_{n, \text { lim }}\right) \\
\left(R_{L C}\right) \\
(11)\end{array}$ \\
\hline J0835-4510 & 36.84 & 11 & 12.53 & 72 & 64 & 1,2 & $1.05-1.06$ & $0.65-0.80$ & 0.25 & 0.23 \\
\hline J0659+1414 & 34.58 & 110 & 12.67 & 29 & 38 & 3 & $1.13-1.14$ & $0.90-1.04$ & 0.30 & 0.60 \\
\hline J0205+6449 & 37.43 & 5 & 12.56 & 78 & 88 & 2 & $0.97-0.98$ & $\cdots$ & 1.00 & 0.71 \\
\hline $\mathrm{J} 2229+6114$ & 37.35 & 11 & 12.31 & 55 & 46 & 2 & $1.01-1.02$ & $\cdots$ & 0.40 & 0.29 \\
\hline J1420-6048 & 37.00 & 13 & 12.38 & 30 & 35 & 6,7 & $1.10-1.11$ & $\ldots$ & 0.50 & 0.42 \\
\hline J2021+3651 & 36.53 & 17 & 12.50 & 75 & 85 & 5 & $0.97-0.98$ & $\cdots$ & 0.40 & 0.40 \\
\hline J1057-5226 & 34.48 & 540 & 12.03 & 75 & 69 & 4 & $0.93-0.94$ & $\ldots$ & 0.10 & 0.20 \\
\hline
\end{tabular}

NOTES.-Col.(1):Pulsar name. Col.(2),(3),(4):The spindown luminosity, the characteristic age and the strength of surface magnetic field, which we adopt in Abdo et al. (2010a). Col.(5):The inclination angle. Col.(6):The viewing angle. Col.(7):Reference for cols.(5) and (6). Col.(8):The emission altitude in the $\gamma$-ray band. Col.(9):The emission altitude in the optical/UV band. Col.(10):Assumed limit of radial distance to null point. Col.(11):Mean free path for $\gamma$-ray photons at the limit of radial distance to the null point. REFERENCES.- (1)Johnston. Karastergiou \& Willett (2006) (2)Ng \& Romani (2008) (3)Everett \& Weisberg (2001) (4)Weltevrede \& Wright (2009) (5)Van Etten. Romani \& Ng (2008) (6)Weltevrede et al. (2010) (7)Weltevrede \& Johnston (2008) 

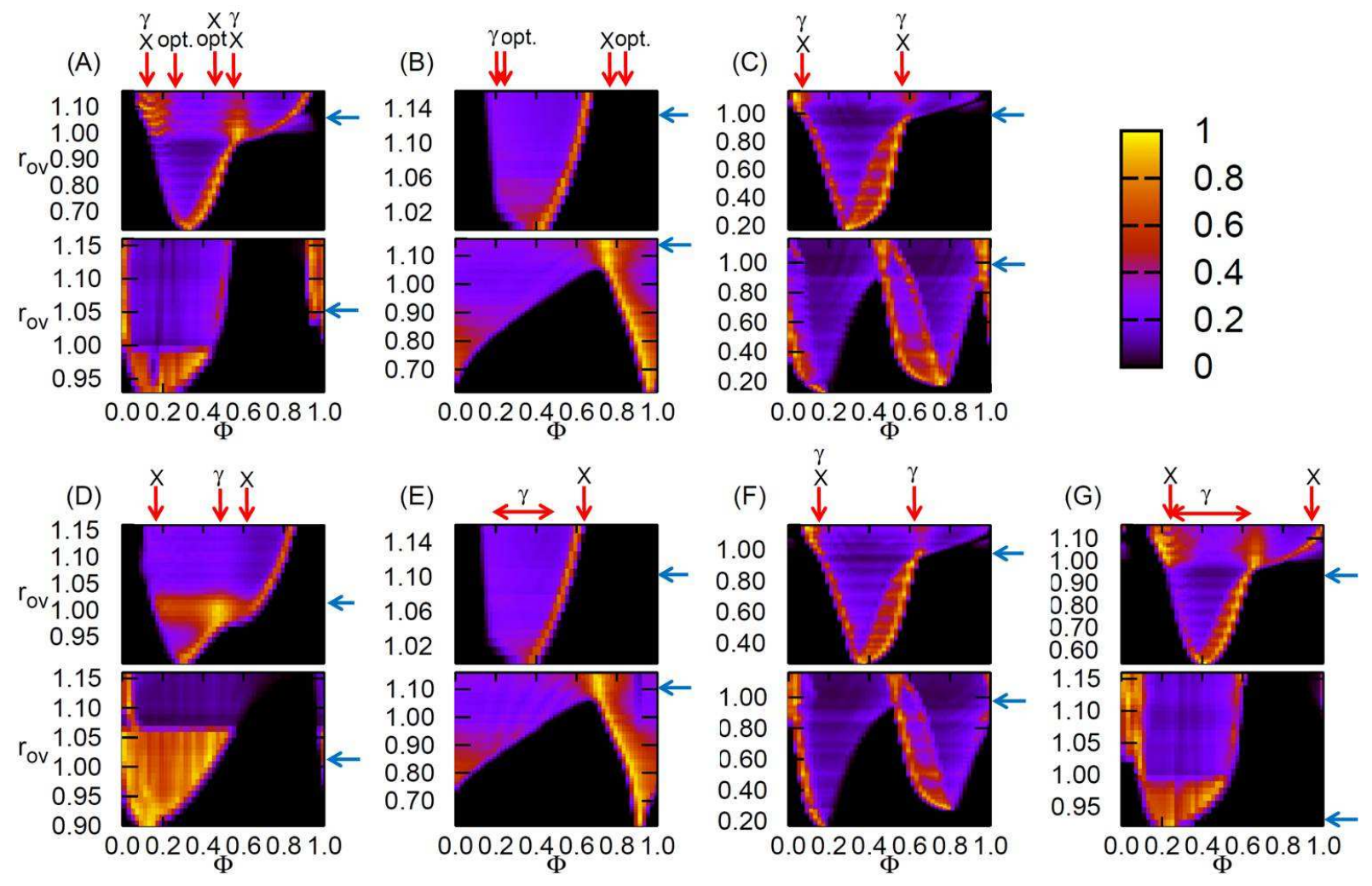

Fig. 1. - The intensity maps for seven pulsars. Upper left to right: PSRs J0835-4510 (A), J0659+1414 (B) and J0205+6449 (C). Lower left to right: PSRs J2229+6114 (D), J1420-6048 (E), J2021+3651 (F) and J1057-5226 (G). In each sample, upper panel is outward emission and lower is inward emission. The blue horizontal arrows show best fit values of $r_{o v}$ for $\gamma$-ray and X-ray emission regions. The red vertical arrows show the phase of peaks. The red horizontal arrows in $(\mathrm{E})$ and $(\mathrm{G})$ show the phase range of broad peaks. 

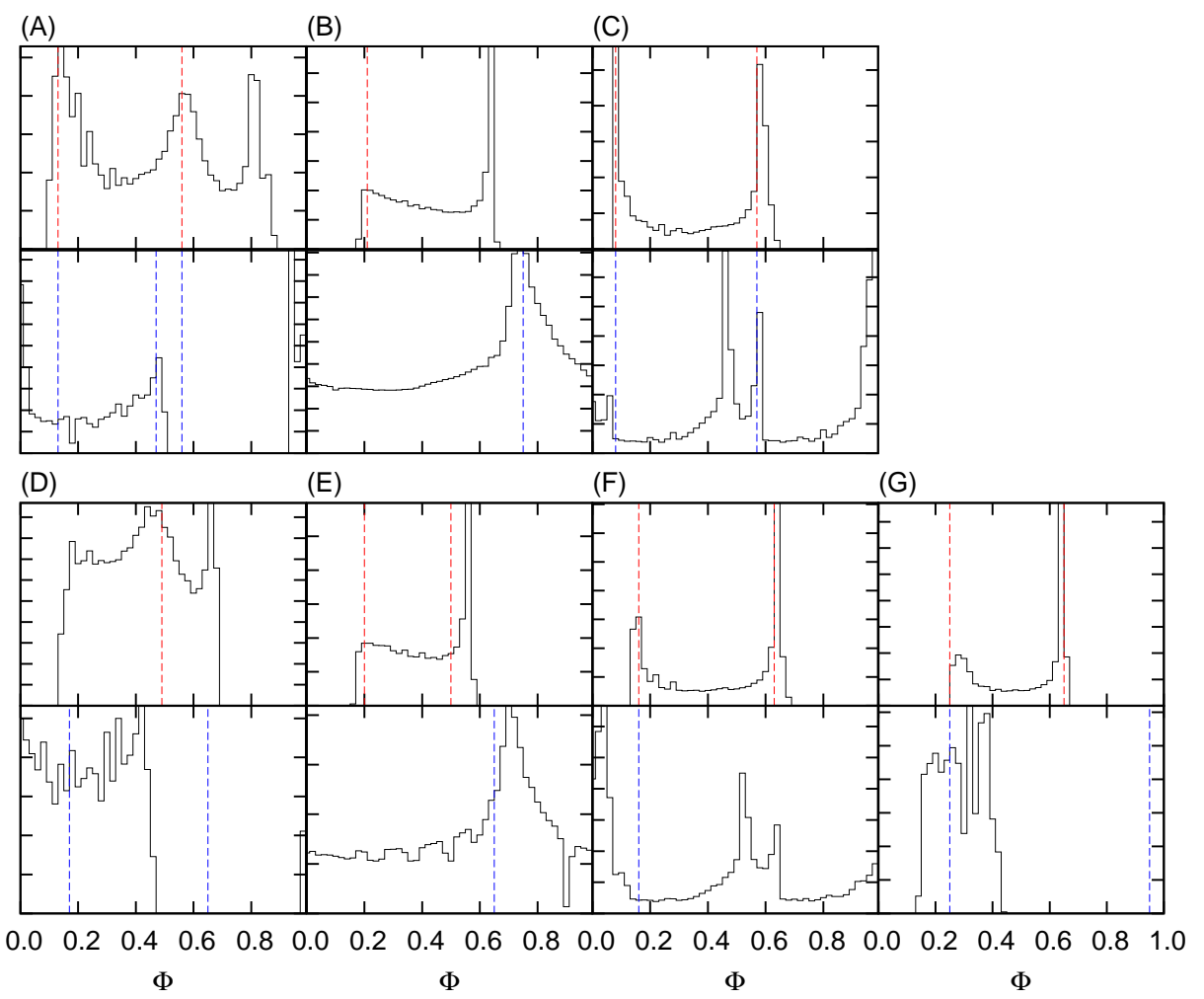

Fig. 2.- The calculated light curves for $\gamma$ - and X-ray emission region. Upper left to right: PSRs J0835-4510 (A), J0659+1414 (B) and J0205+6449 (C). Lower left to right: PSRs J2229+6114 (D), J1420-6048 (E), J2021+3651 (F) and J1057-5226 (G). In each sample, upper panel is outward emission and lower is inward emission. The vertical axis is in arbitrary units. The red and blue short-dashed vertical lines show the phase of $\gamma$-ray and X-ray peaks as in Fig 11. The peaks of PSRs J1420-6048 and J1057-5226 are so broad that the phase range is within two red vertical lines. 

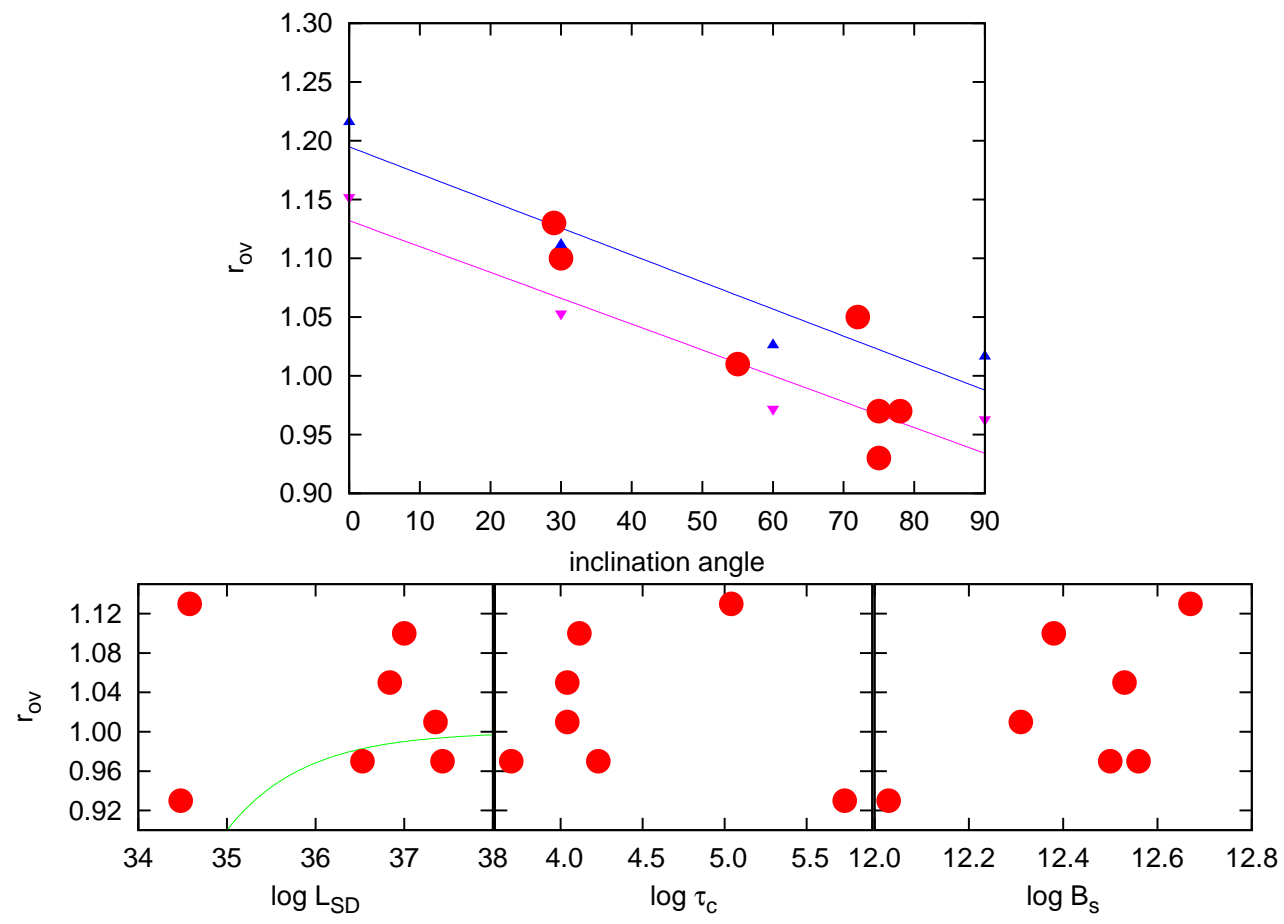

Fig. 3.- The relation between $r_{o v}$ and inclination angle (upper), spin-down luminosity (lower left), characteristic age (lower middle) and surface magnetic field (lower right). The altitudes corresponding to the separatrix layer model are shown as purple downward and blue upward triangles in the upper panel. The two lines are linear fitting lines for the separatrix layer model. The light green curve in the lower left panel shows the relation $\left(1-r_{\text {ov }}\right)=\left(10^{33} \mathrm{ergs}^{-1} / L_{S D}\right)^{1 / 2}$. 


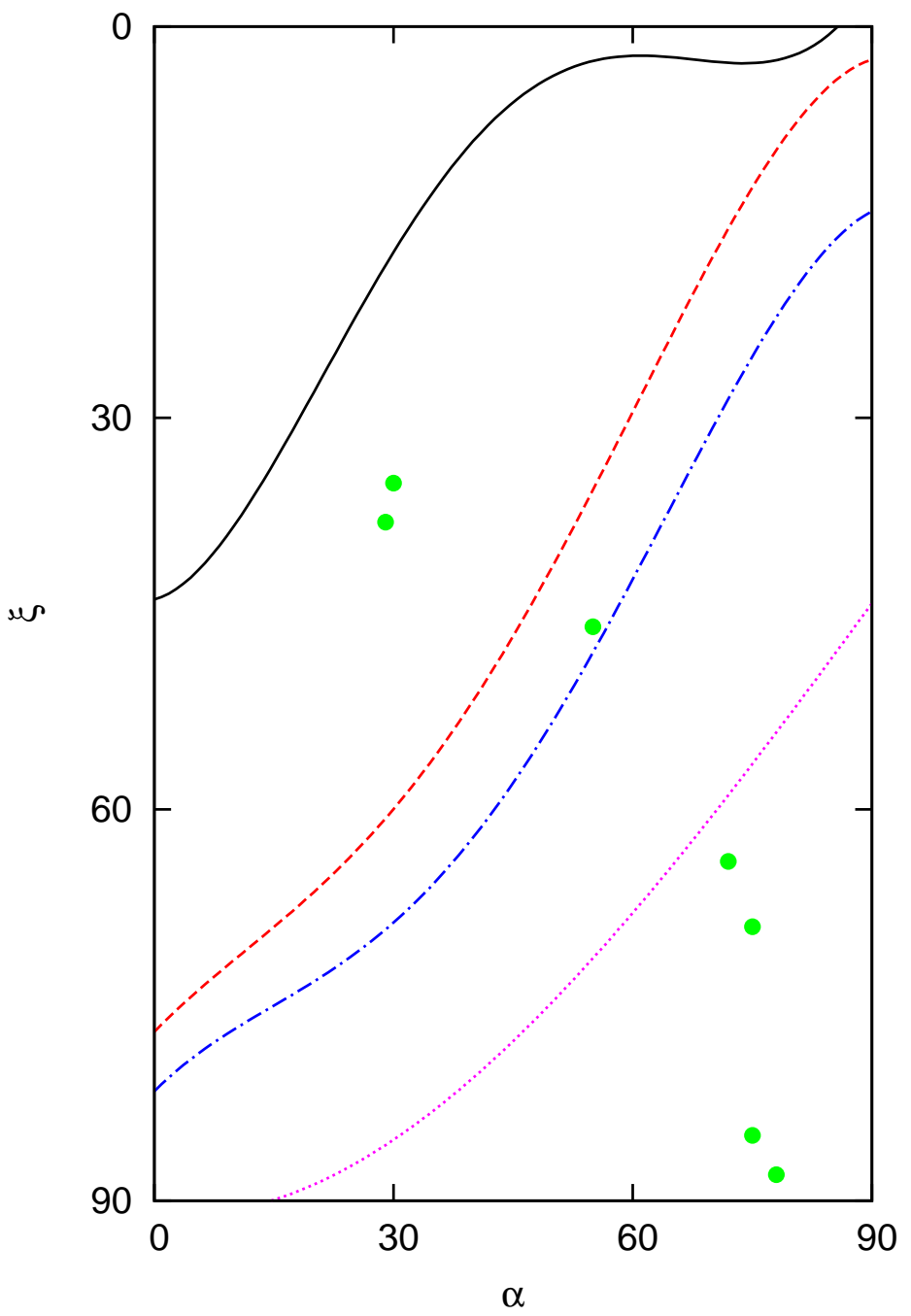

Fig. 4.- The observable range for $\gamma$-ray pulsars in the $\alpha-\xi$ plane for the outer gap model. Black solid curve shows the boundary of observable pulsars using linear fitting line for the separatrix layer model. Red dashed, blue dash-dotted and purple dotted curves show the boundary with $r_{o v}=0.95$, 0.90 and 0.70, respectively. Light-green curcles show the pulsars in Table 1. 


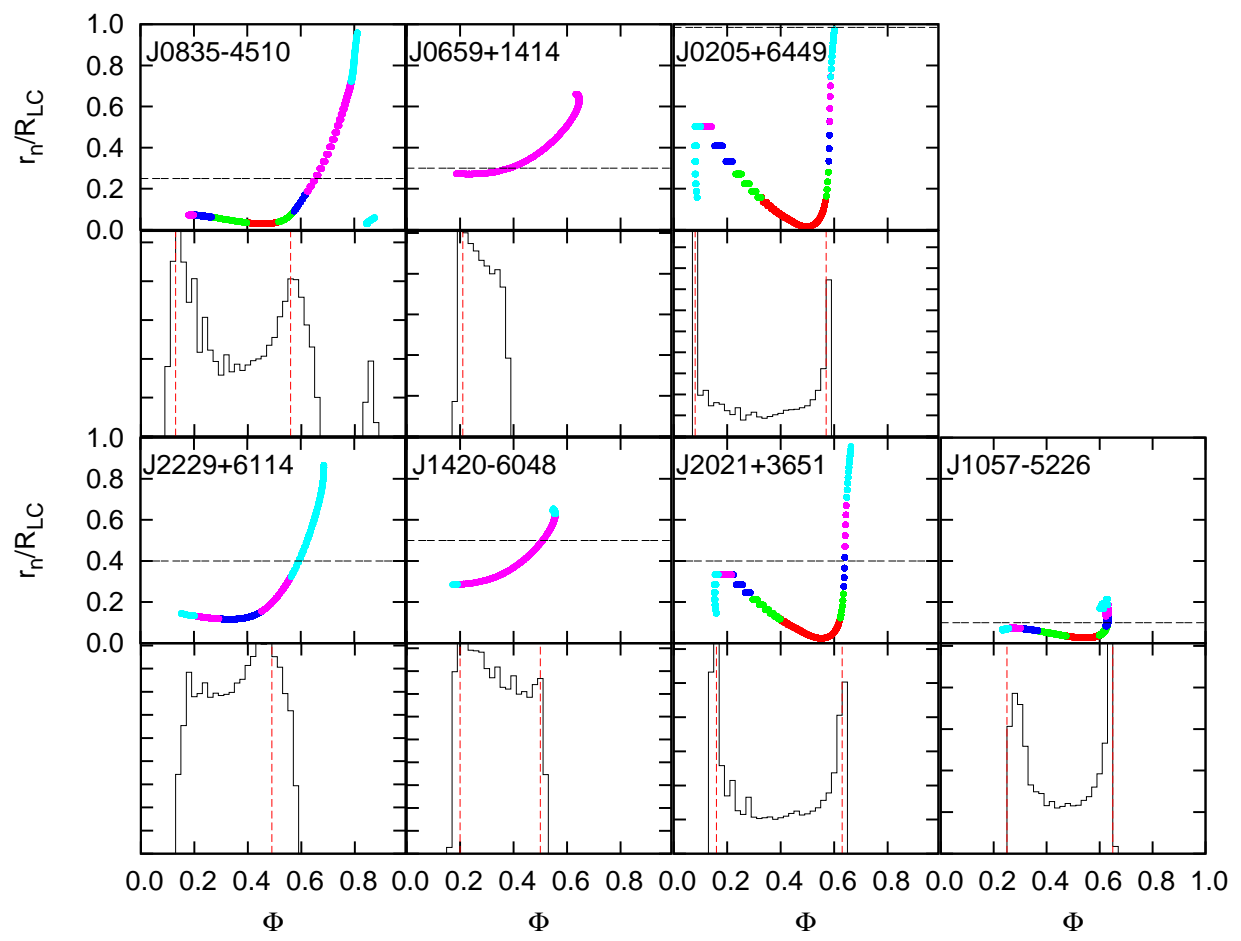

Fig. 5.- The distribution of the radial distance to the null point of the field line on which observed photons are emitted (each upper panel) and the light curves that are restricted by the azimuthal extension limit as a function of the rotation phase (each lower panel) for seven pulsars. The color shows the radial distance to the emitting point as $0.0<r / R_{L C}<0.2$ (red), $0.2<r / R_{L C}<0.4$ (light green), $0.4<r / R_{L C}<0.6$ (blue), $0.6<r / R_{L C}<0.8$ (purple), $0.8<r / R_{L C}<1.0$ (light blue). The values $r_{n, l i m}$ for each pulsar are shown as black long-dashed horizontal lines. The red short-dashed vertical lines show the phases of the $\gamma$-ray peaks. For PSRs J1420-6048 and J1057-5226, the vertical lines show the phase range of broad peaks. 